\title{
Refractory Right Ventricular Failure in a Patient with Emery-Dreifuss Muscular Dystrophy
}

\author{
Yasuyuki Chiba ${ }^{1}$, Arata Fukushima ${ }^{1,2}$, Motoki Nakao ${ }^{1}$, Yuta Kobayashi ${ }^{1}$, Takahiro Ishigaki ${ }^{3}$, \\ Taro Tenma ${ }^{1}$, Kiwamu Kamiya ${ }^{1}$, Yasushige Shingu ${ }^{3}$, Tomonori Ooka ${ }^{3}$, \\ Yoshiro Matsui ${ }^{3,4}$ and Toshihisa Anzai ${ }^{1}$
}

\begin{abstract}
:
A 23-year-old man had progressive muscle weakness and Emery-Dreifuss muscular dystrophy (EDMD) due to a $L M N A$ (lamin A/C) mutation. Congestive heart failure diagnosed at 19 years of age. Maximal drug treatment/cardiac resynchronization failed to improve the cardiac function. He was therefore hospitalized due to heart failure. Despite extracorporeal membrane oxygenation, he developed severe right heart dysfunction and died (multiple organ failure). A cardiac lesion's presence determines the prognosis of EDMD. While there are many arrhythmia reports, few reports on heart failure (particularly severe heart failure requiring cardiac transplantation) have been published. Right heart function monitoring and early ventricular-assist device use plus right heart support considering heart transplantation are important.
\end{abstract}

Key words: right ventricular failure, Emery-Dreifuss muscular dystrophy, left ventricular assist device, heart transplantation

(Intern Med 59: 1277-1281, 2020)

(DOI: 10.2169/internalmedicine.4113-19)

\section{Introduction}

Emery-Dreifuss muscular dystrophy (EDMD) is a hereditary neuromuscular disease characterized by contracture of the elbows, Achilles tendons, and cervical muscles with slowly progressive muscle weakness. These clinical manifestations are caused by the mutation of genes involved in the nuclear envelope proteins emerin and lamin A/C. The clinical manifestation that determines the prognosis of EDMD is a cardiac lesion that starts from the atria, develops to an atrioventricular node, and eventually extends to ventricles. This contributes to atrial arrhythmia, complete atrioventricular block, and progressive ventricular dysfunction, leading to sudden cardiac death and heart failure.

However, the cardiac dysfunction due to EDMD rarely results in severe heart failure that requires heart transplantation. In addition, there are a few documented cases in which right ventricular (RV) failure is progressive and leads to a critical condition, even after the introduction of mechanical circulatory support, such as a left ventricular assist device (LVAD) and extracorporeal membrane oxygenation (ECMO).

We herein report a case of EDMD with progressive RV failure that was not controlled by the maximal dose of medications or by cardiac resynchronization therapy (CRT). The RV failure was refractory even under the support of veno-arterial (VA) ECMO, leading to the patient's death due to multiple organ failure, including liver failure. Our patient's case highlights the importance of the close monitoring of the RV function and the consideration of the early introduction of a biventricular assist device (BiVAD) including right heart support or heart transplantation in EDMD patients with laminopathies.

\footnotetext{
${ }^{1}$ Department of Cardiovascular Medicine, Faculty of Medicine and Graduate School of Medicine, Hokkaido University, Japan, ${ }^{2}$ Asabu Heart and Gastrointestinal Clinic, Japan, ${ }^{3}$ Department of Cardiovascular and Thoracic Surgery, Faculty of Medicine and Graduate School of Medicine, Hokkaido University, Japan and ${ }^{4}$ Hanaoka Seishu Memorial Cardiovascular Clinic, Japan

Received: October 30, 2019; Accepted: December 24, 2019; Advance Publication by J-STAGE: February 19, 2020

Correspondence to Dr. Arata Fukushima, arating77@huhp.hokudai.ac.jp
} 


\section{Case Report}

A 23-year-old Japanese man was admitted to our hospital due to worsening exertional dyspnea. He had shown difficulty running at two years old and been diagnosed with EDMD with a LMNA (Lamin A/C) gene mutation at exon 1 from leucine 102 to proline. In accord with the typical EDMD manifestations, his elbows, knees, and Achilles tendons had become contracted at five years old, and his posterior cervical muscles became rigid; however, he continued to go about his usual daily life, albeit with mild exercise restriction. His father had also been diagnosed with EDMD and died suddenly in his $40 \mathrm{~s}$.

When the patient was 19 years old, he was admitted to a hospital for the first time due to congestive heart failure. Signs of RV failure, such as leg edema and liver congestion, were prominent, as indicated by the high blood bilirubin level $(2.5 \mathrm{mg} / \mathrm{dL})$. Echocardiography showed that the tricuspid annular plane systolic excursion (TAPSE) was $5.7 \mathrm{~mm}$, RV-s' $5.1 \mathrm{~cm} / \mathrm{s}$, and RV fractional area change (RVFAC) $19 \%$, indicating marked RV dysfunction. High-dose diuretics in addition to carvedilol (10 mg/day) and enalapril $(2.5 \mathrm{mg} /$ day) were introduced. Despite those medications, he was readmitted due to worsening RV failure.

At 22 years old, an implantable cardioverter defibrillator (ICD) was implanted due to his non-sustained ventricular tachycardia (VT) and family history of sudden cardiac death. At this point, right heart catheterization (RHC) showed a pulmonary artery wedge pressure (PAWP) of 22 $\mathrm{mmHg}$, right atrial pressure (RAP) of $17 \mathrm{mmHg}$, and right ventricular stroke work index (RVSWI) of 6.47, suggesting sustained severe RV dysfunction. As such, RV failure was likely to be the main pathological condition throughout his clinical course.

He had symptomatic heart failure with New York Heart Association class III under maximal doses of guidelinedirected medication, and his electrocardiogram showed a regular sinus rhythm and left bundle branch block with wide QRS (150 ms). His LV ejection fraction (LVEF) was $\leq 35 \%$ on echocardiography. He was therefore considered to be indicated for CRT and was thus upgraded from ICD at 23 years old.

On admission, his blood pressure was 106/65 $\mathrm{mmHg}$, and his heart rate was 60 beats/min with a regular rhythm. The physical examination revealed a third cardiac sound and pan-systolic murmur (Levine II/VI) at the apical region. Respiratory sounds were clear, whereas a distended jugular vein and lower-limb edema were evident. Chest X-ray indicated an increased cardiothoracic ratio (59\%) with no pulmonary congestion (Fig. 1A).

The electrocardiogram showed all biventricular pacing due to CRT (Fig. 1B). Echocardiography revealed mild dilatation in both ventricles (LV end-diastolic diameter: $54 \mathrm{~mm}$, RV diameter $50 \mathrm{~mm}$ ) with a reduced LVEF (30\%). The RV function was also diminished, as indicated by a decreased
RVFAC $(11 \%)$ and a low TAPSE $(8 \mathrm{~mm})$. Both enlarged ventricles caused leaflet tethering and coaptation loss that resulted in moderate mitral regurgitation and severe tricuspid regurgitation. The inferior vena cava was dilated to $23 \mathrm{~mm}$ with reduced respiratory fluctuation.

In the histological findings of posterior cervical muscles performed later at the autopsy, increased variation in muscle fiber size with moderate infiltration of connective tissue was observed, which was compatible with EDMD (Fig. 1D). Blood tests revealed brain natriuretic peptide (BNP) 196.1 $\mathrm{pg} / \mathrm{mL}$, total bilirubin $1.4 \mathrm{mg} / \mathrm{dL}$, aspartate aminotransferase (AST) $39 \mathrm{U} / \mathrm{L}$, alanine aminotransferase (ALT) $43 \mathrm{U} / \mathrm{L}$, and $\gamma$-glutamyl transpeptidase $(\gamma$-GTP) $451 \mathrm{U} / \mathrm{L}$, which suggested liver dysfunction. Although the coronary arteries were intact on angiography, an RV endocardial biopsy revealed cardiac hypertrophy with increased magnitudes of the nucleus and myocardium.

Electron microscopy demonstrated typical findings of EDMD: a reduced number of myofibrils, necrosis, and the formation of pseudo- or true inclusion. Since other secondary cardiomyopathies were ruled out by these clinical and histological findings, we diagnosed EDMD-related cardiomyopathy.

The patient's clinical course is shown in Fig. 2. RHC on day 11 showed that the cardiac index $(\mathrm{CI})$ was $2.0 \mathrm{~L} / \mathrm{min} / \mathrm{m}^{2}$ (Fick method), and PAWP was $15 \mathrm{mmHg}$, indicating Forrester subset IV. The pulmonary artery pressure (PAP) was 29/12 (18) $\mathrm{mmHg}$, and the RAP was $15 \mathrm{mmHg}$. The RVSWI, one of the hemodynamic parameters for the RV function, was calculated with the following formula: RVSWI $\left(\mathrm{g} \cdot \mathrm{m} / \mathrm{m}^{2} /\right.$ beat $)=[$ mean pulmonary artery pressure $(\mathrm{mPAP})$ mean right atrial pressure (mRAP)] $\times$ stroke volume index (SVI) $\times 0.0136$. His RVSWI was $1.36 \mathrm{~g} \cdot \mathrm{m} / \mathrm{m}^{2} /$ beat, indicating severe RV dysfunction. To compensate for the patient's low output syndrome and congestion, we started a continuous dobutamine $(3.0 \mu \mathrm{g} / \mathrm{kg} / \mathrm{min})$ infusion and added milrinone $(0.125 \mu \mathrm{g} / \mathrm{kg} / \mathrm{min})$ on day 16 .

On day 32, the RHC data were improved, with an increased CI (2.48 L/min $\left./ \mathrm{m}^{2}\right)$, reduced PAWP (13 $\mathrm{mmHg}$ ), and increased RVSWI $\left(2.8 \mathrm{~g} \cdot \mathrm{m} / \mathrm{m}^{2} /\right.$ beat $)$. However, on day 38 , the patient's renal function progressively worsened, possibly due to high central venous pressure (CVP) and hypotension because the blood pressure had been around 70/40 $\mathrm{mmHg}$ several days before the worsening of the renal function. To increase his blood pressure, a continuous dopamine $(2.0 \mu \mathrm{g} /$ $\mathrm{kg} / \mathrm{min}$ ) infusion was initiated; the milrinone was reduced, and enalapril and eplerenone were discontinued. In addition, intra-aortic balloon pumping (IABP) and continuous renal replacement therapy (CRRT) were temporarily needed. As the patient's hemodynamics improved in response to increase mixed venous oxygen saturation $\left(\mathrm{SvO}_{2}, 70-80 \%\right)$ and CI (3.0-3.5 L/min $\left./ \mathrm{m}^{2}\right)$, the IABP and CRRT were withdrawn on day 45 .

On day 68 , however, the CI dropped to $1.25 \mathrm{~L} / \mathrm{min} / \mathrm{m}^{2}$ again, and the patient's lung congestion and fluid retention deteriorated; the PAWP was as high as the RAP, at 22 
A

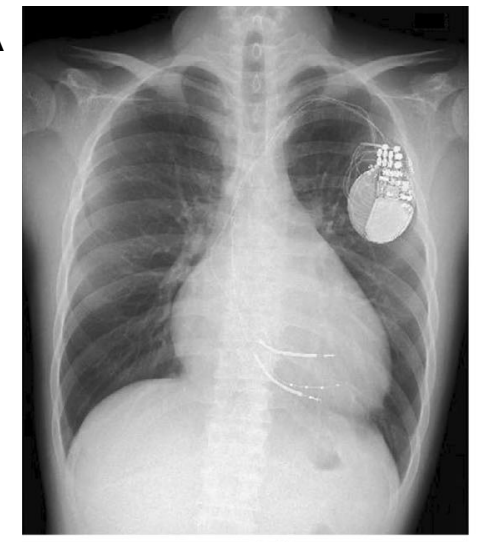

C

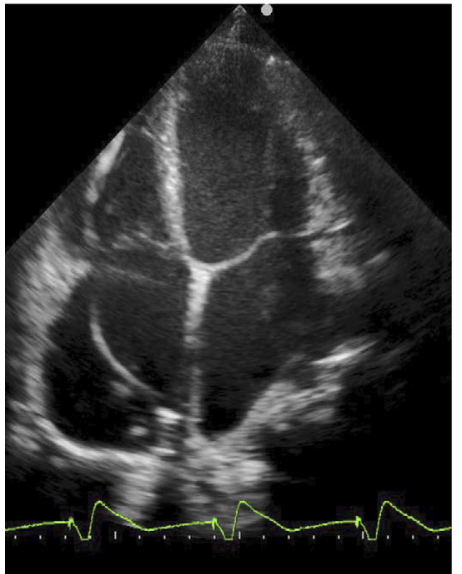

B

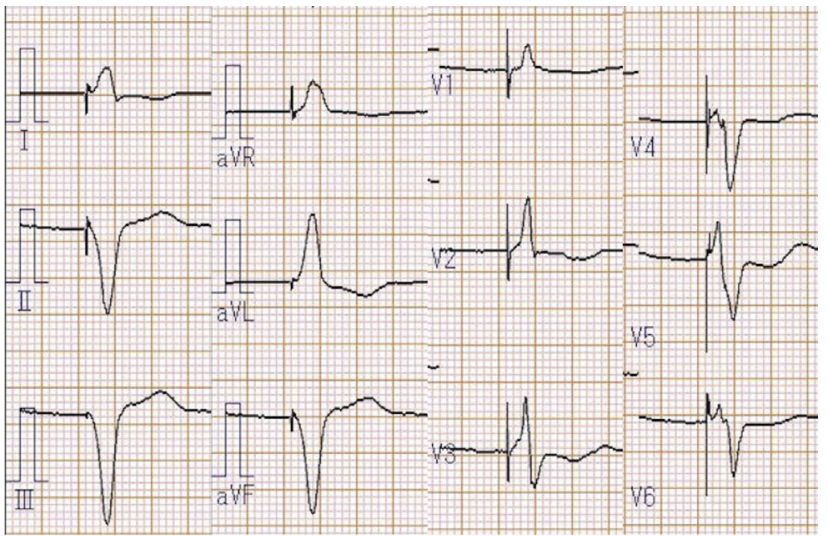

D

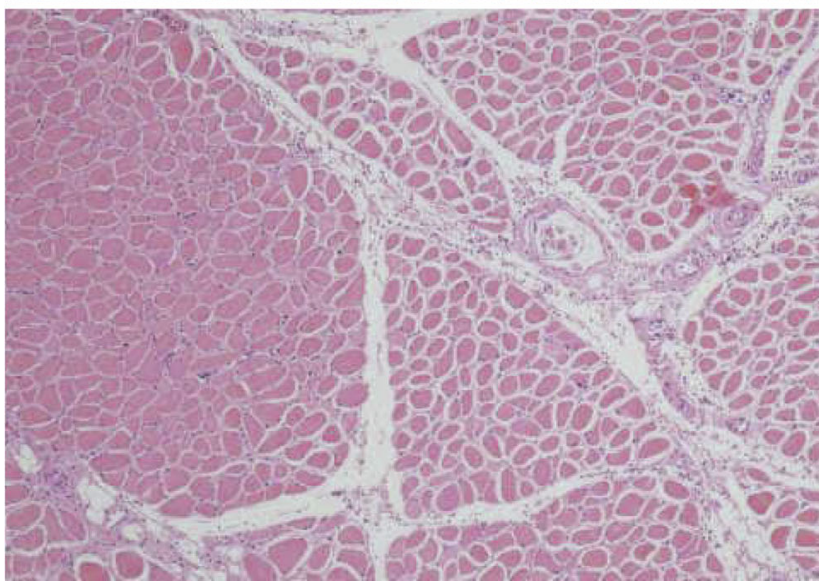

Figure 1. A: Chest $\mathrm{X}$-ray showing the increased cardiothoracic ratio (59\%) with no pulmonary congestion. B: An electrocardiogram showing all biventricular pacing due to CRT. C: Echocardiography indicating marked LV/RV dilation and dysfunction. D: Histological findings of the posterior cervical muscles at the autopsy showing increased variation in the muscle fiber size with moderate infiltration of connective tissue, distinctive of Emery-Dreifuss muscular dystrophy. CRT: cardiac resynchronization therapy, LV: left ventricular, $\mathrm{RV}$ : right ventricular

mmHg. Furthermore, the patient's serum bilirubin levels and liver transaminase dramatically increased, suggesting the development of liver failure. On day 71, VA ECMO through a femoral vein to the subclavian artery was initiated, but due to the systemic hemorrhagic diathesis accompanied by the disseminated intravascular coagulation (DIC), the patient died of multiple organ failure on day 100.

An autopsy performed under informed consent from the patient's family revealed efferent enlargement in both ventricles with a thin wall thickness (Fig. 3A). The histopathological examination revealed that mild to moderate myocardial hypertrophy and severe fibrosis were evident in the RV (Fig. 3B, C) and LV (Fig. 3D). The weight of the liver was significantly increased to $2,298 \mathrm{~g}$, and macroscopic liver congestion and cholestasis were detected. On microscopic images, the portal vein area was scarcely changed, and atrophy or loss of hepatocytes was observed around the central veins, indicating "shock liver." In the lungs, extensive alveolar hemorrhaging was observed, and a collection of macrophages had ingested hemosiderin was noted, reflecting systemic hemorrhagic diathesis.

\section{Discussion}

Our patient's case exhibited the conduction disturbance commonly seen in EDMD, and his condition progressed to $\mathrm{RV}$-dominant heart failure. The RV failure induced systemic congestion, acute liver failure, and resultant systemic hemorrhagic diathesis despite ECMO support, leading to the patient's death due to multiple organ failure. Although cardiac involvement is a common comorbidity and a major prognostic factor for EDMD, few patients with EDMD-related cardiomyopathy develop heart failure that requires heart transplantation $(1,2)$. Indeed, Boriani, et al. reported that only $5.5 \%$ of EDMD patients underwent heart transplantation (3).

The diversity of such cardiac lesions may be due to the differences in gene mutations that code nuclear envelope proteins in EDMD. Two different inherent types have been documented in EDMD: an X-linked recessive inheritance pattern (EDMD1) and an autosomal-dominant mutation (EDMD2). The cardiac lesion of EDMD2 is known to manifest with more severe complications (e.g., ventricular arrhythmias and dilated cardiomyopathy) at younger ages than EDMD1. This phenotype in EDMD2 is related to the muta- 


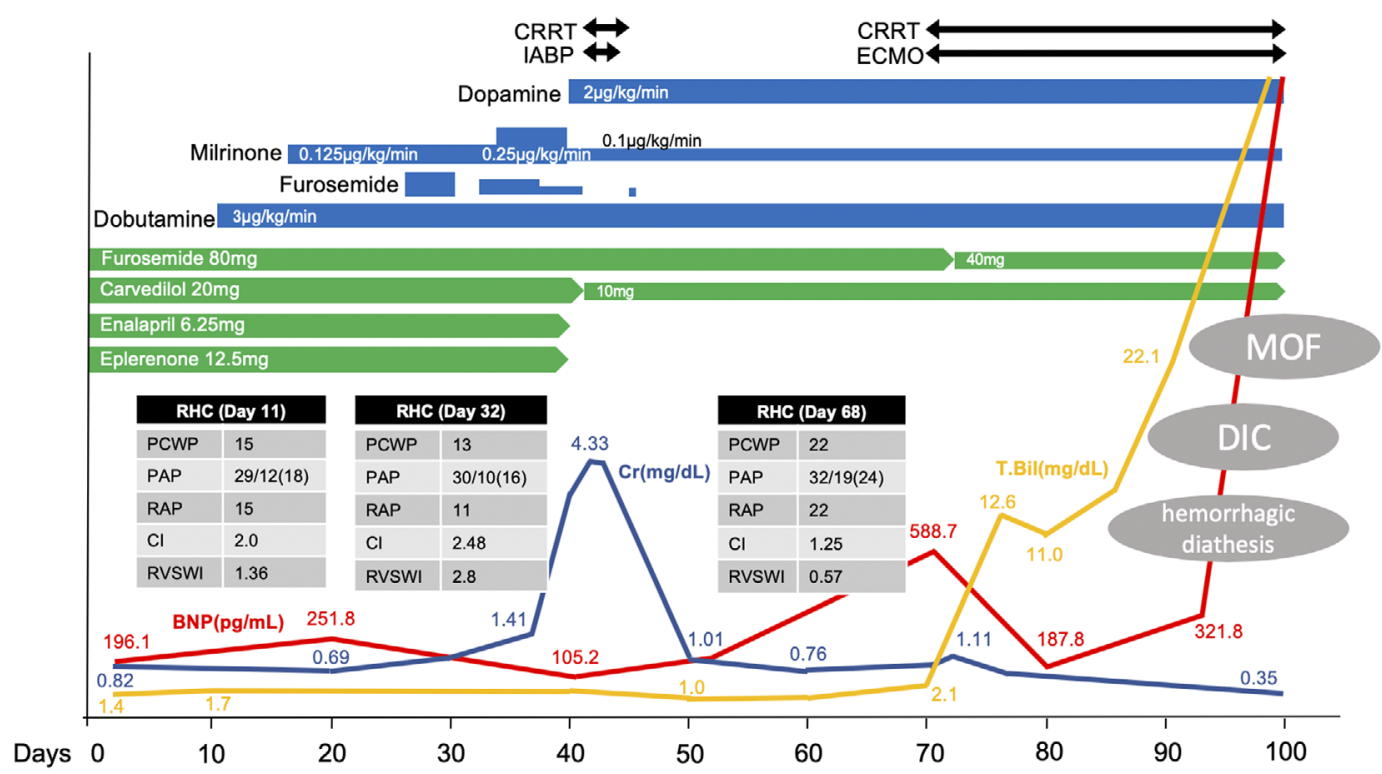

Figure 2. The patient's clinical course. Right heart catheterization (RHC) on day 11 showed severe LV/RV dysfunction and congestion, and some inotropes were started. Although the patient's hemodynamics improved, his renal function progressively worsened on day 38 . Concerned about the worsening of his renal function due to the blood pressure decline, dopamine and mechanical support (IABP and CRRT) were initiated, and the patient's renal function and hemodynamics improved. However, he developed acute liver failure along with severe RV failure indicated by the RHC data on day 68. Although veno-atrial ECMO was introduced on day 71, liver dysfunction and DIC caused systemic hemorrhagic diathesis, and the patient died of multiple organ failure on day 100. CRRT: continuous renal replacement therapy, DIC: disseminated intravascular coagulation, ECMO: extracorporeal membrane oxygenation, IABP: intra-aortic balloon pumping, LV: left ventricle, RV: right ventricle

A

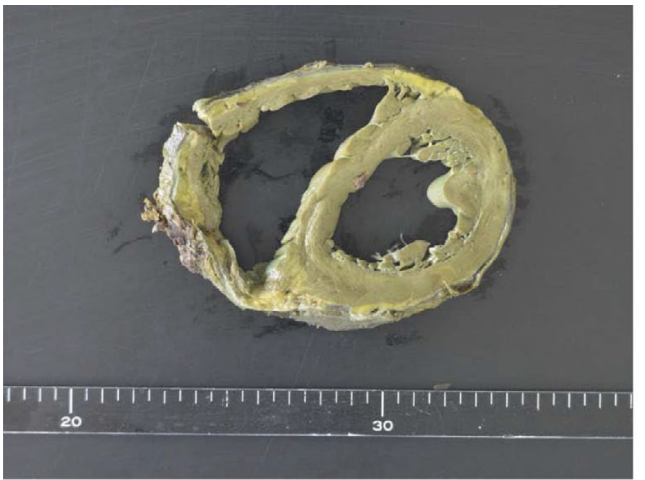

C

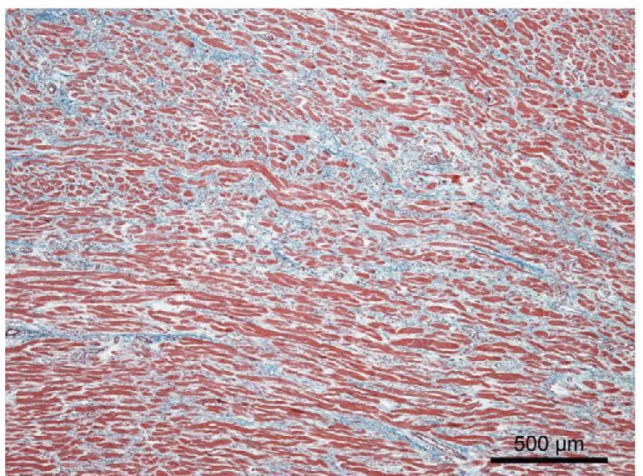

B

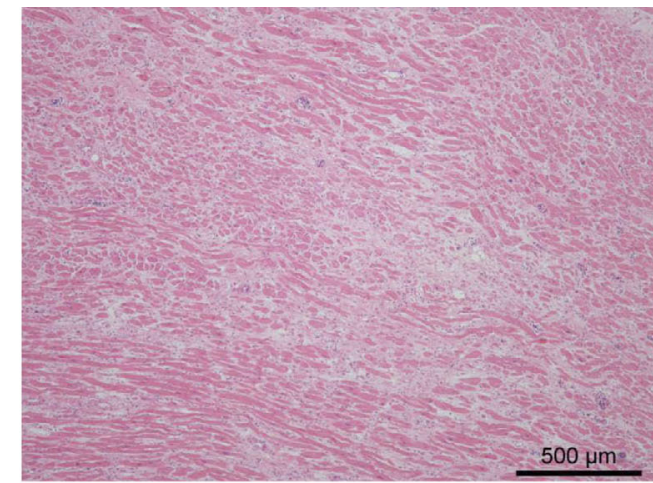

D

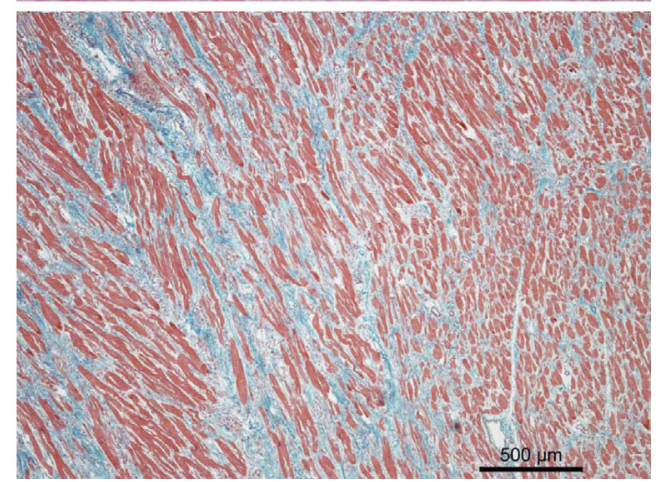

Figure 3. Pathological findings of the patient's heart. A: Macroscopic image indicating enlargement of both ventricles with thin wall thickness. B: Microscopic images of the right ventricular myocardium with Hematoxylin and Eosin staining showing mild to moderate myocardial hypertrophy. Masson's trichrome staining demonstrating extensive interstitial fibrosis between the myocardium in both the RV (C) and LV (D). 
tion in the LMNA gene encoding lamin $\mathrm{A}$ and $\mathrm{C}$, which are components of the nuclear envelope (4).

Notably, LMNA mutations are known to account for $6-8 \%$ of dilated cardiomyopathies with conduction defect or ventricular arrhythmia, which are associated with a high risk of sudden cardiac death $(5,6)$. These arrhythmias can be the earliest clinical manifestation prior to the development of cardiac chamber dilatation. Thus, the LMNA mutation should be considered when the premature conduction system abnormality manifests together with unexplained dilated cardiomyopathy. Although such a conduction disease requires the implantation of a permanent pacemaker (PPM), an ICD is often necessary to prevent ventricular arrhythmia and sudden death in these patients. However, the prophylactic application of ICD in patients with LMNA mutations should be further discussed. LMNA mutations are also found in $4 \%$ of patients with arrhythmogenic right ventricular cardiomyopathy (ARVC) (7). Such LMNA mutations with diverse phenotypes are now recognized as laminopathies, and EDMD is also considered to represent the adult onset of these laminopathies.

The features of cardiomyopathy associated with laminopathy are dilation of the LV and RV, ventricular noncompaction, and poor clinical outcome. As such, the biventricular failure, especially the progressive RV failure in the present case, may be attributed to the ventricular remodeling underpinned by laminopathies.

Our patient had a low RVSWI, high CVP, and high PTINR, all indicating the risk of RV failure after LVAD implementation (8). Although additional RVAD might be needed, the reported 1-year survival rate after BiVAD transplantation is $<50 \%$, which is much worse than that of LVAD alone (9). We therefore decided to manage this patient by VA-ECMO and then planned to conduct LVAD (not BiVAD), but his RV failure could not be controlled, and the resultant liver failure and systemic hemorrhagic diathesis were fatal. Furthermore, the timing of LVAD or heart transplantation was apparently too late, as it took six months to determine the effect of CRT on the patient's heart failure, which was resistant to the maximal dose of medications.

To date, there have been only a few case reports on the benefits of CRT for muscular dystrophy, such DMD and $\mathrm{BMD}$, and no reports concerning its efficacy in EDMD have been published (10). Our patient also showed extensive cardiac fibrosis in the LV and RV, suggesting the limited efficacy of CRT. Indeed, it was challenging to evaluate the cardiac fibrosis before the autopsy in the present case because cardiac magnetic resonance imaging (the gold standard for the evaluation for cardiac fibrosis) could not be performed due to the patient's post-ICD implantation status. The earlier introduction of BiVAD or heart transplantation might have been needed to save his life, given the high prevalence of VT and progressive HF in cases of laminopathy.

\section{Conclusion}

Patients with EDMD with an LMNA mutation may develop severe biventricular dysfunction as well as conduction disturbance that is refractory even after CRT-D implantation and ECMO. Close monitoring of the RV function in patients with laminopathies is essential to determine the optimal timing to introduce further interventions, such as BiVAD or heart transplantation.

The authors state that they have no Conflict of Interest (COI).

\section{References}

1. Feingold B, Mahle WT, Auerbach S, et al. Management of cardiac involvement associated with neuromuscular diseases: a scientific statement from the American Heart Association. Circulation 136: e200-e231, 2017.

2. Nakajima K, Aiba T, Makiyama $\mathrm{T}$, et al. Clinical manifestations and long-term mortality in lamin $\mathrm{A} / \mathrm{C}$ mutation carriers from a Japanese multicenter registry. Circ J 82: 2707-2714, 2018.

3. Boriani G, Gallina M, Merlini L, et al. Clinical relevance of atrial fibrillation/flutter, stroke, pacemaker implant, and heart failure in Emery-Dreifuss muscular dystrophy: a long-term longitudinal study. Stroke 34: 901-908, 2003.

4. Wang S, Peng D. Cardiac involvement in Emery-Dreifuss muscular dystrophy and related management strategies. Int Heart J 60: 12-18, 2019.

5. Fatkin D, MacRae C, Sasaki T, et al. Missense mutations in the rod domain of the lamin A/C gene as causes of dilated cardiomyopathy and conduction-system disease. N Engl J Med 341: 1715-1724, 1999.

6. Tobita T, Nomura S, Fujita T, et al. Genetic basis of cardiomyopathy and the genotypes involved in prognosis and left ventricular reverse remodeling. Sci Rep 8: 1998, 2018.

7. Quarta G, Syrris P, Ashworth M, et al. Mutations in the Lamin A/ $\mathrm{C}$ gene mimic arrhythmogenic right ventricular cardiomyopathy. Eur Heart J 33: 1128-1362, 2012.

8. Bellavia D, Iacovoni A, Scardulla C, et al. Prediction of right ventricular failure after ventricular assist device implant: systematic review and meta-analysis of observational studies. Eur J Heart Fail 19: 926-946, 2017.

9. Kirklin JK, Naftel DC, Pagani FD, et al. Seventh INTERMACS annual report: 15,000 patients and counting. J Heart Lung Transplant 34: 1495-1504, 2015.

10. Walker S, Levy T, Rex S, Paul VE. Biventricular implantable cardioverter defibrillator use in a patient with heart failure and ventricular tachycardia secondary to Emery-Dreifuss syndrome. Europace 1: 206-209, 1999.

The Internal Medicine is an Open Access journal distributed under the Creative Commons Attribution-NonCommercial-NoDerivatives 4.0 International License. To view the details of this license, please visit (https://creativecommons.org/licenses/ by-nc-nd/4.0/).

(C) 2020 The Japanese Society of Internal Medicine

Intern Med 59: 1277-1281, 2020 